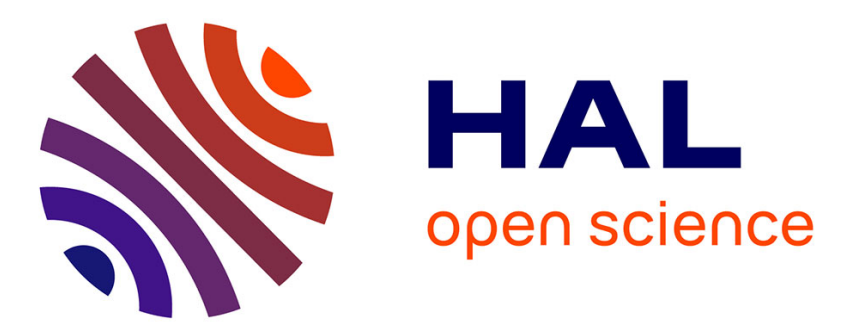

\title{
Etude par visualisations ultra rapides et mesures de pression d'une source impulsionnelle large bande non-directive
}

\author{
Philippe Pernod, A. Merlen, A. Kemmou, A. Ahyi
}

\section{To cite this version:}

Philippe Pernod, A. Merlen, A. Kemmou, A. Ahyi. Etude par visualisations ultra rapides et mesures de pression d'une source impulsionnelle large bande non-directive. Journal de Physique IV Proceedings, 1994, 04 (C5), pp.C5-729-C5-732. 10.1051/jp4:19945157 • jpa-00252836

\section{HAL Id: jpa-00252836 https://hal.science/jpa-00252836}

Submitted on 1 Jan 1994

HAL is a multi-disciplinary open access archive for the deposit and dissemination of scientific research documents, whether they are published or not. The documents may come from teaching and research institutions in France or abroad, or from public or private research centers.
L'archive ouverte pluridisciplinaire HAL, est destinée au dépôt et à la diffusion de documents scientifiques de niveau recherche, publiés ou non, émanant des établissements d'enseignement et de recherche français ou étrangers, des laboratoires publics ou privés. 


\title{
Etude par visualisations ultra rapides et mesures de pression d'une source impulsionnelle large bande non-directive
}

\author{
Ph. PERNOD, A. MERLEN*, A. KEMMOU et A. AHYI
}

Institut d'Electronique et de Micro-électronique du Nord, Département Opto-Acousto-Electronique (IEMN-DOAE), UMR 9929 du CNRS, Ecole Centrale de Lille, BP. 48, 59651 Villeneuve d'Ascq cedex, France

* Laboratoire de Mécanique de Lille, Equipe Mécanique des Fluides, LML, URA 1441 du CNRS, Université des Sciences et Technologies de Lille, Cité Scientifique, Bâtiment M3, 59655 Villeneuve d'Ascq cedex, France

\begin{abstract}
Ponctual sound sources with very short and simple signature (the ideal beeing a diractype) could be very usefull in all the fields of transient acoustics. However, such sources cannot be realised by conventionnal electroacoustic transducers, because these last cannot be considered as point sources and generally have poor transient responses. We present here the solution we have developped, a "mini-sparker" which generates a very brief acoustic impulse. The characterization of the wave emitted is performed both, by ultra-rapid cinematographic ombroscopic visualisations, and broad-banded pressure measurements.
\end{abstract}

\section{INTRODUCTION}

Dans un grand nombre de domaines de 1'acoustique impulsionnelle (CND, Acoustique Médicale, Acoustique Sous-Marine...), il serait intéressant de disposer d'une source ponctuelle, de signature courte et simple (type dirac). Citons par exemple la caractérisation des capteurs acoustiques, l'identification de cibles, la modélisation physique d'explosions sous-marines ou de phénomènes sismiques (modèle réduit $[1,2]$ ), les études de cavitation transitoire...

De telles sources sont difficilement réalisables à l'aide des transducteurs électroacoustiques conventionnels : en effet ces derniers ne peuvent pas être considérés comme des sources ponctuelles, et ont, en général, une réponse transitoire médiocre.

Nous présentons dans le présent article la solution originale que nous avons développée et qui utilise une décharge électrique haute tension dans l'eau (mini-étinceleur). Le rayonnement de cette source, ainsi que les phénomènes mécaniques accompagnant la décharge sont étudiés simultanément par ombroscopie ultra-rapide et mesures de pression.

\section{DISPOSITIFS EXPERIMENTAUX}

Le schéma de principe de notre source acoustique impulsionnelle (mini-étinceleur) est présenté Figure 1.a : la source est constituée d'un condensateur à décharge rapide de $18 \mathrm{nF}$ chargé par une source haute tension $(1 \mathrm{kV}$ à $10 \mathrm{kV})$, d'un thyratron jouant le rôle d'interrupteur commandant la décharge, et de deux électrodes coaxiales espacées de $0,5 \mathrm{~mm}$. Deux géométries de l'extrêmité du support d'électrodes ont été utilisées, l'une cônique, l'autre cylindrique.

La caractérisation du rayonnement de cette source a été réalisée selon le dispositif expérimental de la Figure $1 \mathrm{~b}:$ l'onde impulsionnelle est émise dans un aquarium d'eau déminéralisée $\left(30 \times 15 \times 30 \mathrm{~cm}^{3}\right)$, le système coaxial d'électrodes étant disposé verticalement quelques centimètres sous la surface du liquide. Un hydrophone large bande $(100 \mathrm{kHz}-10 \mathrm{MHz} \pm 6 \mathrm{~dB}$, sens. $-243 \mathrm{~dB}$ re $1 \mathrm{~V} / \mu \mathrm{Pa}$ [3]) relié à un oscilloscope numérique à mémoire (Lecroy 9410) enregistre le signal reçu à une dizaine de centimètres sous la source. Simultanément, une cinématographie ombroscopique ultrarapide, basée sur la technique de Cranz-Schardin [4], est utilisée pour la visualisation du front d'onde émis et les phénomènes qui lui succèdent (cavitation, émissions secondaires). Le système, synchronisé avec la génération de l'onde acoustique impulsionnelle (le retard étant 

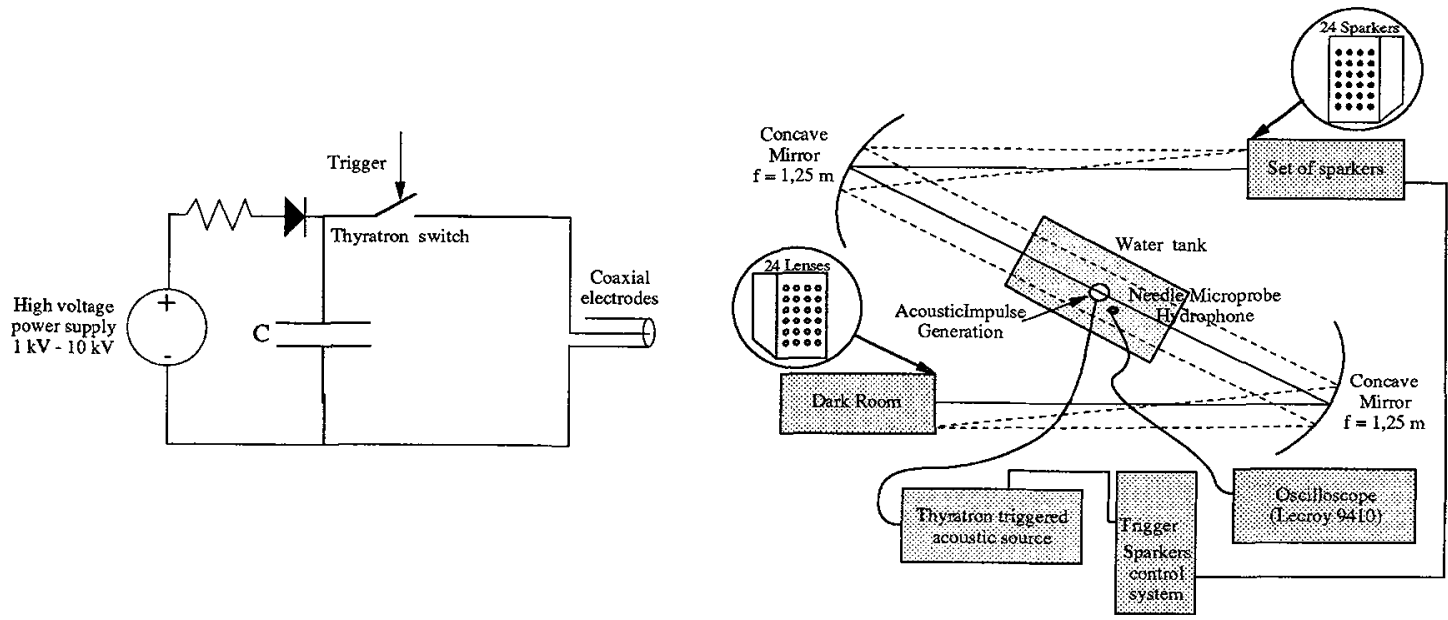

Figure 1 : (a) Schéma de principe de la source mini-étinceleur; (b) Dispositif de visualisation ombroscopique de type Cranz-Schardin ( 24 images à cadence variable de 1 image à 10 millions d'images par secondes).

réglable), émet une séquence de 24 éclairs successifs de durée $300 \mathrm{~ns}$, à intervalles de temps réglables de $0,1 \mu \mathrm{s}$ à $1 \mathrm{~s}$ ( 1 image à 10 millions images / sec). Les faisceaux successifs sont rendus parallèles pour la traversée du champ d'analyse, puis refocalisés pour donner 24 images du phénomène sur une plaque photographique $(18 \mathrm{x}$ $24 \mathrm{~cm}^{2}$ ).

\section{RESULTATS}

La description des phénomènes mis en auvre dans le fonctionnement de notre source peut être effectuée à partir des visualisations par ombroscopie et mesures de pression des figures 2 à 7 :

Une première série de 3 photographies est extraite des 24 obtenues lors d'une prise d'images toutes les $3 \mu$ s (retard 0). Elle présente l'onde primaire émise par la source (figure 2a). L'onde émise fait apparaître un front impulsionnel bien marqué, sensiblement sphérique, suivi d'oscillations concentriques d'amplitudes plus faibles. Ces dernières sont probablement dues aux résonnances du système d'électrodes, mais tendent à disparaître au fur et à mesure de la progression de l'onde. S'il est possible qu'au tout début de sa génération l'onde soit un choc, elle devient très vite acoustique comme le montre la figure 2 b déduite des visualisations, et où la vitesse mesurée est constante $(1458 \mathrm{~m} / \mathrm{s})$ quelques microsecondes après l'émission.

Une deuxième série de photos à cadence plus lente que la précédente $(R=0 \mu \mathrm{s}, \Delta t=40 \mu \mathrm{s})$ visualise les instants suivant l'émission de l'onde pimaire (figure 3). On y voit l'apparition et la première oscillation d'une bulle de cavitation résultant de la perturbation du milieu par l'arc électrique. La durée totale de l'oscillation est de $800 \mu \mathrm{s}$, les phases de croissance et de décroissance de la bulle étant symétriques. La taille maximale atteinte à $\mathrm{t}=400 \mu \mathrm{s}$ est de $9 \mathrm{~mm}$ suivant l'axe horizontal, et $7 \mathrm{~mm}$ suivant l'axe vertical. Les photos font par ailleurs clairement apparaître les réflexions multiples de l'onde primaire sur les parois de la cuve, la courbure du front d'onde s'ammenuisant. A la fin de son cycle, la bulle implose et entamme une nouvelle oscillation de plus faible amplitude tout en s'éloignant du système d'électrodes. Simultanément à ce rebond, une nouvelle onde estémise (figure 3 dernière photo et figure $4: \mathrm{R}=730 \mu \mathrm{s}, \Delta \mathrm{t}=5 \mu \mathrm{s}$ ). Cette onde possède des caractéristiques semblables à celles de l'onde primaire mais les résonnances du système d'électrodes ont cette fois disparues, la bulle s'étant désolidarisée de la source. Jusqu'à trois oscillations de bulles ont ainsi été observées dans nos expériences, chacune se ponctuant par l'émission d'une nouvelle onde. Dans la dernière oscillation nous avons constaté que la bulle perd en général la régularité de sa forme. Dans certains cas, elle se sépare en plusieurs bulles plus petites comme dans le cas de la figure 5 où deux ondes quasi-simultannées centrées chacunes sur leur bulle d'origine sont émises.

L'allure temporelle de l'onde primaire observée à $10 \mathrm{~cm}$ de la source par l'hydrophone large bande est présentée figure 6 (conditions de décharge : Condensateur $\mathrm{C}=18 \mathrm{nF}$, Tension de charge $\mathrm{V}=8 \mathrm{kV}$ ). L'impulsion 
(a)

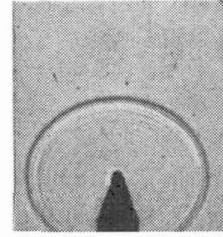

$6 \mu \mathrm{s}$

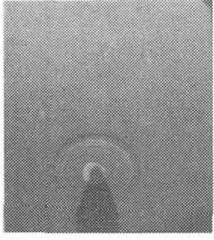

$12 \mu \mathrm{s}$

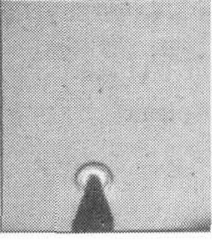

$24 \mu \mathrm{s}$ (b)

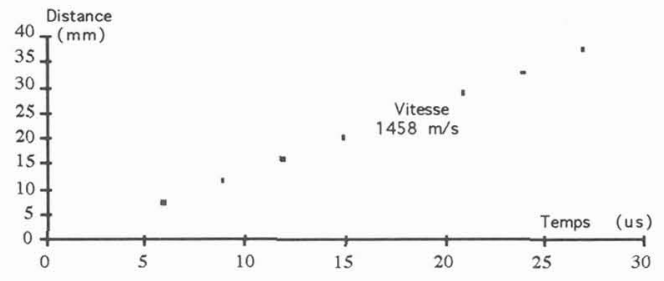

Figure 2 : (a) Visualisation de l'onde primaire ( 3 photos extraites parmi les 24 obtenues lors de l'acquisition); (b) Position du front d'onde fonction du temps mesurée à partir de la cinématographie.

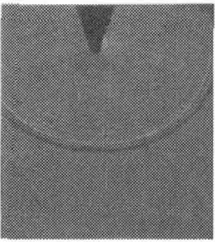

$40 \mu \mathrm{s}$

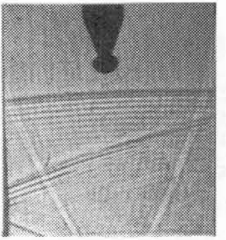

$320 \mu \mathrm{s}$

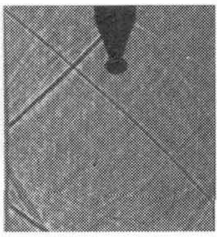

$600 \mu \mathrm{s}$

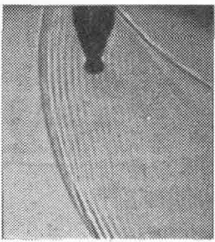

$120 \mu \mathrm{s}$

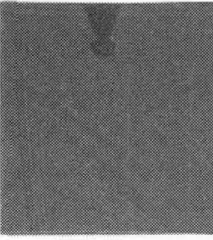

$400 \mu \mathrm{s}$

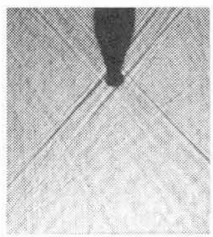

$680 \mu \mathrm{s}$

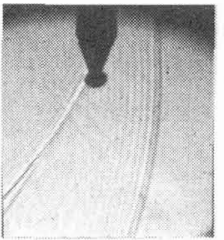

$200 \mu \mathrm{s}$

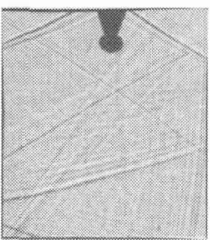

$480 \mu \mathrm{s}$

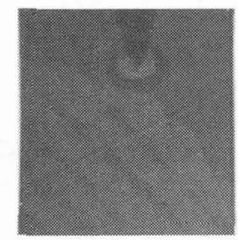

$800 \mu \mathrm{s}$
Figure 3 : Première oscillation de la bulle de cavitation faisant suite à l'onde primaire visible sur la première photo de la séquence.

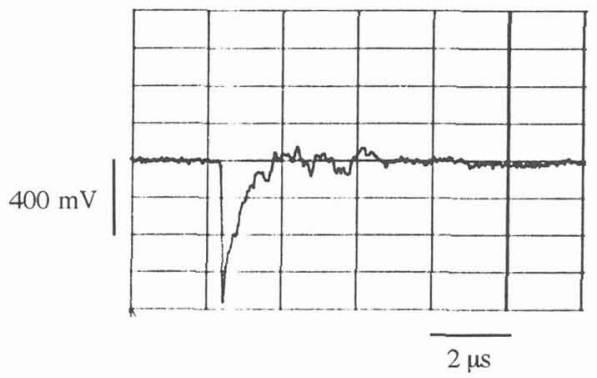

Figure 6 :Allure temporelle de l'onde primaire enregistrée par un hydrophone large bande $[100 \mathrm{kHz}$ $10 \mathrm{MHz}$, sensibilité $-243 \mathrm{~dB}$ re $1 \mathrm{~V} / \mu \mathrm{Pa}$ ]

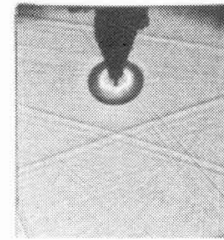

$755 \mu \mathrm{s}$

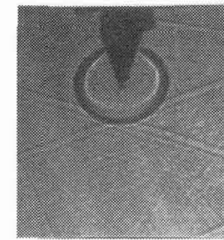

$760 \mu \mathrm{s}$

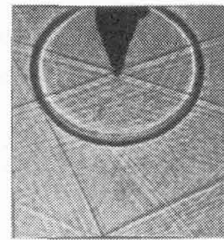

$770 \mu \mathrm{s}$
Figure 4 : Visualisation de l'onde secondaire générée au rebond de la bulle de cavitation (Comparable à dernière photo de la figure 3 ).

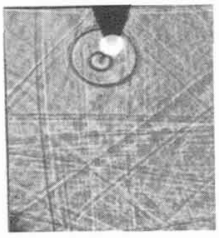

$1100 \mu \mathrm{s}$

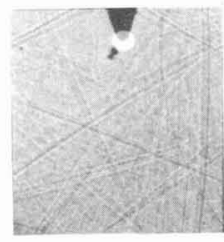

$1180 \mu \mathrm{s}$
Figure 5 : Fin de la $2^{\text {ème }}$ oscillation de la bulle de cavitation : la bulle s'est séparée en deux, et deux "troisièmes" ondes sont générées. (a)

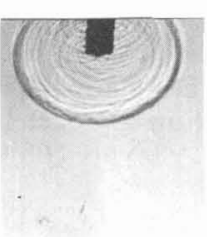

$20 \mu \mathrm{s}$ (b)

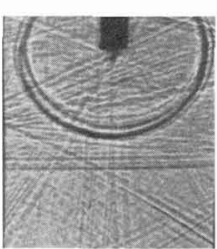

$22 \mu \mathrm{s}$
Figure 7 : Ondes obtenues pour le support d'électrodes cylindrique : (a) Onde primaire ; (b) Onde secondaire. 
produite est constituée d'un front de montée très raide de durée $75 \mathrm{~ns}$ au cours duquel la pression passe de 0 à 10,3 bars, suivi d'une décroissance exponentielle plus lente, la largeur totale de l'impulsion étant de 1,6 $\mu \mathrm{s}$. L'amplitude négative présentée par le signal provient de la polarisation de l'hydrophone.

La géométrie du support d'électrodes a une grande importance pour les caractéristiques de l'onde rayonnée. La figure 7 présente à titre d'exemple les ondes primaires et secondaires obtenues dans le cas d'un support cylindrique. La diffraction à l'émission ainsi que les résonnances de la partie mécanique sont bien observées. Il y a dans ce cas dégradation de l'allure temporelle de l'onde primaire.

Afin de compléter l'étude expérimentale précédente, nous avons cherché à préciser les relations déterminant les principaux paramètres acoustiques du rayonnement de notre source. Uneétude théorique complète des phénomènes étant extrêmement complexe, nous avons utilisé une approche par similitude. Suite à nos observations, nous nous sommes restreints au cas de l'approximation de l'acoustique, pesanteur et viscosité étant négligées, et aucune longueur n'étant supposée intervenir dans le problème. Ainsi, nous avons puécrire le temps $\tau$ séparant onde primaire et onde secondaire, et la valeur maximale de la pression en un point donné, en fonction des trois grandeurs primaires $\rho_{0}$ (densité statique), $\mathrm{p}_{0}$ (pression statique), et $\mathrm{E}$ (énergie mécanique supposée apportée instantanément au milieu au moment de la décharge électrique) :

$$
\tau=\mathrm{k}_{1} \frac{\mathrm{E}_{\frac{1}{3}} \rho_{0}^{\frac{1}{2}}}{\mathrm{p}_{0}^{\frac{5}{6}}} \quad \mathrm{p}_{\max }=\mathrm{k}_{2} \frac{\mathrm{E}_{\frac{1}{3}} \mathrm{p}_{0}^{\frac{2}{3}}}{\mathrm{r}} \quad ; \text { soit : } \mathrm{p}_{\max }=\frac{\mathrm{k}_{2}}{\mathrm{k}_{1}} \frac{\mathrm{p}_{0}^{\frac{3}{2}}}{\mathrm{r} \rho_{0}^{\frac{1}{2}}} \tau
$$

Il est intéressant de noter la dépendance en $\mathrm{E}^{1 / 3}$ de ces deux quantités. Ainsi, si l'on était capable de déterminer les constantes $\mathrm{k}_{1}$ et $\mathrm{k}_{2}$, il serait aisément possible de déduire de la mesure de $\tau$ ou de $\mathrm{p}_{\max }$ l'énergie mécanique de la source, et par suite son rendement. C'est ce que nous envisageons de faire dans la suite de nos travaux, une optimisation du rendement étant probablement possible. Nous noterons simplement encore ici que le rapport des deux relations précédentes fait apparaître une relation de linéarité entre la pression maximale obtenue en un point et le temps séparant les deux ondes primaires et secondaires, cette relation est indépendante de $\mathrm{E}$. Les mesures expérimentales de pression couplées aux visualisations, nous ont permis de vérifier cette relation de linéarité et d'en déduire le rapport $\mathrm{k}_{2} / \mathrm{k}_{1}$ dans la configuration étudiée.

\section{CONCLUSION}

Une source ponctuelle large bande de signature acoustique courte et simple a été mise au point. Une description détaillée de ses caractéristiques a été rendue possible par l'utilisation d'un système de visualisation ombroscopique ultra-rapide, couplé à l'utilisation d'un hydrophone large bande de petites dimensions. Grâce à cette association, nous disposons à présent d'un outil nous permettant le développement de nombreuses applications d'acoustique impulsionnelle, et en particulier, de faire dans un premier temps des mesures expérimentales de réponses impulsionnelles de capteurs et de cibles, ou d'étudier l'interaction d'une onde de choc avec des particules en mouvement dans un écoulement. Nous envisageons également l'amélioration de notre système de visualisation ultra-rapide afin d'aboutir à des prișes d'images quantitatives, par interférométrie ou holographie, avec acquisition numérique d'images.

\section{REFERENCES}

[1] Pernod P., Piwakowski B., Delannoy B., Tricot J.C., "Detection of shallow underground cavities by seismic methods : physical modelling approach", Acoustical Imaging, edited by Hiroshi Shimizu \&al. Tohoku University Sendaï Japan June 1988, pp. $705-713$.

[2] Pernod P., Piwakowski B., Tricot J.C., Delannoy B., Pierre J.M., "Détection d'objets diffractants à faible profondeurs par méthodes sismiques : modélisation physique", Premier Congrès Français d'Acoustique, Supplément au n 2 du Journal de Physique, Lyon France Avril 1990 (Les Editions de Physique, Les Ulis).

[3] Nakamura Y., Otani T., "Frequency response of a piezoelectric polymer film hydrophone and an elastic wave induced on the backing surface", Proceedings of 12th Symposium on Ultrasonics Electronics, Tokyo 1991.

[4] Cranz C., Schardin H., Z. Phys., 56, 147 (1929). 